\title{
Asymptotic stability analysis and error estimate for a class of shallow water wave equation *
}

\author{
Zhang Jun \\ (School of Mathematics and Statistical,Guizhou University of Finance and Econmics, Guiyang 550025,China)
}

\begin{abstract}
In this paper, the asymptotic stability and numerical method of shallow water wave equation with Benjamin-Bona-Mahony type is considered. Under suitable assumption, we prove that the solution of the shallow water wave equation is asymptotically convergent to the steady-state solution of the equation, and some exponentially decay rate are obtained. In addition, we also construct a numerical scheme of the equation, we prove that the scheme is unconditionally stable, and we also get the estimate of the full discrete scheme. Finally, some results of the theoretical analysis are verified by numerical experiments
\end{abstract}

Key words : BBM type equation, asymptotic stability analysis, exponential decay, unconditional stability, error estimate

\section{$\S 1$ Introduction}

Since Benjamin et al.[1] proposed the Benjamini-Bona-Mahony(BBM) model in 1972, the research of this kind of model has been a hot topic. The latest research shows that this kind of model can be used to describe the wave propagation process from deep water to shallow water. The theoretical and numerical research of this kind of model has attracted the interest of researchers.

Medeiros et al.[2] studied the existence and uniqueness of solutions to BBM equation. Amick et al. [3] investigated the long-term behavior of the solution of BBM equation, they used energy estimation, maximum principle, and Cole-Hopf transformation to get the decay rate result of the solution:

$$
\|u(\cdot, t)\|_{L^{2}(\mathbb{R})}=O\left(t^{-\frac{1}{4}}\right), \quad\left\|\partial_{x} u(\cdot, t)\right\|_{L^{2}(\mathbb{R})}=O\left(t^{-\frac{3}{4}}\right),\|u(\cdot, t)\|_{L^{\infty}(\mathbb{R})}=O\left(t^{-\frac{1}{2}}\right) .
$$

Biler [4] considered the generalized two-dimensional BBM equation and obtained some estimates of the decay rate:

$$
\|u\|_{L^{\infty}}=O\left(t^{-\frac{1}{3}}\right),\|u\|_{L^{\infty}}=O\left(t^{-\frac{2}{3}}\right) .
$$

Mei[5] used Fourier transform method and point-by-point method of Green's function to obtain the decay rate estimates for the BBM equation. Chen et

\footnotetext{
* Corresponding author at: School of Mathematics and Statistical,Guizhou University of Finance and Econmics, Guiyang 550025,China. E-mail: zj654440@163.com
} 
al.[6] studied the two-dimensional small amplitude water wave model and obtained some estimation results of the attenuation rate of some solutions. Zhang [7], Guo et al.[8] obtained the decay rate of BBM equation solution in highdimensional space by using low-frequency Fourier method and high-frequency energy method. In the recent research, Kundu et al. [9] obtained the asymptotic attenuation estimation results of BBM equation with homogeneous boundary and the error estimation of some numerical solutions. Numerical aspects: Omrani et al. $[10,11]$ given a second-order numerical scheme for solving BBM equation. The time direction was discretized by Crank-Nicol scheme, and the space direction was discretized by standard finite element method, and the corresponding error estimate was obtained. Dogan [13] used finite element method to solve a class of regular long wave equations, for very small amplitude waves, the algorithm has good accuracy for small amplitude waves. Qin [14] given a numerical scheme for solving BBM equations by using fully discrete mixed finite element method.

In this paper, we will study the asymptotic convergence property and numerical scheme of a class of shallow water wave models, First, we assume that the steady-state equation has a minimum eigenvalue, and by using the energy estimation method, we prove that the solution of BBM shallow-water wave equation converges gradually to the solution of the steady-state equation, and we also get the exponential decay rate estimates under different norms. We also study the numerical format of the equation, i.e. time is discretized by Crank - Nicol Son method, space is discretized by Fourier-Galerkin method, the convergence order of the scheme is $\mathcal{O}\left(\Delta t^{2}+N^{1-m}\right)$, here $\Delta t$ is the time discrete step size, $N$ is polynomial order, $m$ is the smoothness of the solution. Finally, we give some numerical examples to verify the correctness of the theoretical analysis.

The structure of this paper is as follows: In the second section, the asymptotic stability analysis of the equation will be given. The third section will discuss the time semi-discrete format. The fourth section will analyze the error estimation of the fully discrete format. Finally, we will give some numerical results.

\section{$\S 2 \quad$ Asymptotic stability analysis}

We consider the following dissipative BBM shallow water wave equation:

$$
\partial_{t} u+\partial_{x} u-\partial_{x}^{2} \partial_{t} u+u \partial_{x} u-\nu \partial_{x}^{2} u=f(x, t), t \in(0, \infty), x \in R,
$$

satisfy the following initial conditions:

$$
u(x, 0)=u_{0}(x), \quad x \in R,
$$

and boundary conditions:

$$
u(x, t)=u(x+L, t), \quad t \in(0, \infty), x \in R,
$$

Here $\nu$ is a non-negative constant, $\partial_{x}^{2} \partial_{t} u$ is the dispersion term, $\partial_{x}^{2} u$ is the dissipative term. , $f(x, t) \mathrm{A}$ is a given function.

Here we study the asymptotic properties of the solution of the equation(1)(3) when $t \rightarrow \infty$. Suppose $\lim _{t \rightarrow \infty} u(x, t)=u^{\infty}$, here $u^{\infty}$ is the steady state solution of the equation, then

$$
\begin{aligned}
& \partial_{x} u^{\infty}+u^{\infty} \partial_{x} u^{\infty}-\nu \partial_{x}^{2} u^{\infty}=f^{\infty}, x \in R, \\
& u^{\infty}(x)=u^{\infty}(x+L), x \in R,
\end{aligned}
$$


where $f^{\infty}(x)=\lim _{t \rightarrow \infty} f(x, t)$.

Consider the weak form of the steady state equation (4)-(5)

$$
\left(\partial_{x} u^{\infty}, v\right)+\left(u^{\infty} \partial_{x} u^{\infty}, v\right)+\nu\left(\partial_{x} u^{\infty}, \partial_{x} v\right)=\left(f^{\infty}, v\right), \forall v \in H_{*}^{1} .
$$

Now we consider the following assumptions:

(A1) Eigenvalue problem

$$
\partial_{x} \phi+\partial_{x} u^{\infty} \phi-\nu \partial_{x}^{2} \phi=\lambda \phi, \quad \phi(x)=\phi(x+L),
$$

Has the smallest positive eigenvalue $\lambda_{0}>0$. observe $\forall \phi \in H^{2} \cap H_{*}^{1}$, then

$$
\int_{0}^{L} \partial_{x} u^{\infty} \phi^{2} d x+\nu\left\|\partial_{x} \phi\right\|_{0}^{2}=\lambda\|\phi\|_{0}^{2} \geq \lambda_{0}\|\phi\|_{0}^{2} .
$$

As can be seen from the above equation

$$
\begin{aligned}
\nu\left\|\partial_{x} \phi\right\|_{0}^{2}+\left(u^{\infty} \partial_{x} \phi+\partial_{x} u^{\infty} \phi, \phi\right) & =\nu\left\|\partial_{x} \phi\right\|_{0}^{2}+\frac{1}{2} \int_{0}^{L} \partial_{x} u^{\infty} \phi^{2} d x \\
& \geq \frac{\lambda_{0}}{2}\|\phi\|_{0}^{2}+\frac{\nu}{2}\left\|\partial_{x} \phi\right\|_{0}^{2} \geq \frac{\nu}{2}\left\|\partial_{x} \phi\right\|_{0}^{2} .
\end{aligned}
$$

Using the above inequalities and assumptions A1, we can obtain the existence and uniqueness of solutions to the equation (4)-(5).

Poincaréinequality: $\forall \psi \in H_{*}^{1}(\Omega)$, then $\|\psi\| \leq \frac{1}{\sqrt{\lambda_{1}}}\left\|\partial_{x} \psi\right\|$, here $\lambda_{1}=\left(\frac{2 \pi}{L}\right)^{2}$ is the first minimum eigenvalue of homogeneous Dirichlet eigenvalue problem

$$
\begin{aligned}
& -\partial_{x}^{2} \psi=\lambda \psi, \\
& \psi(x)=\psi(x+L) .
\end{aligned}
$$

Based on this, we have the following estimation results of steady-state solutions.

Lemma 2.1 Let $u^{\infty}$ be the solution of (4)-(5), The following estimate holds:

$$
\begin{aligned}
\left\|\partial_{x} u^{\infty}\right\| & \leq C\left\|f^{\infty}\right\|_{-1}, \\
\left\|u^{\infty}\right\| & \leq C\left\|f^{\infty}\right\|_{-1}, \\
\left\|u^{\infty}\right\|_{L^{\infty}} & \leq C\left\|f^{\infty}\right\|_{-1} .
\end{aligned}
$$

Proof: Taking the inner product with $u^{\infty}$, we have

$$
\nu\left\|\partial_{x} u^{\infty}\right\|_{0}^{2}=\left(f^{\infty}, u^{\infty}\right) \leq C\left\|f^{\infty}\right\|_{-1}\left\|\partial_{x} u^{\infty}\right\|_{0} .
$$

Then we obtain (9). Using Poincaréinequality, we have

$$
\left\|u^{\infty}\right\|_{0} \leq \frac{1}{\sqrt{\lambda_{1}}}\left\|\partial_{x} u^{\infty}\right\|_{0} \leq C\left\|f^{\infty}\right\|_{-1} .
$$

That is

$$
\left\|u^{\infty}\right\|_{L^{\infty}}^{2} \leq C\left\|u^{\infty}\right\|_{0}\left\|\partial_{x} u^{\infty}\right\|_{0} \leq C\left\|f^{\infty}\right\|_{-1}^{2} .
$$

Here, we will give the asymptotic stability results, that is, we will prove that the difference between the solution of equation (1)-(3) and the solution of steady state equation (4)-(5) is asymptotically convergent with respect to time $t$. 
First, let $z=u-u^{\infty}$, then combine with (1)-(3)and (4)-(5), we have

$$
\begin{aligned}
& \partial_{t} z-\partial_{t} \partial_{x}^{2} z+\partial_{x} z-\nu \partial_{x}^{2} z+u^{\infty} \partial_{x} z+z \partial_{x} u^{\infty}=F, \quad x \in R, t>0, \\
& z(x, 0)=u_{0}(x)-u^{\infty}(x)=z_{0}, \\
& z(x, t)=z(x+L, t),
\end{aligned}
$$

here $F=f-f^{\infty}$.

The weak form of equation (12)-(14) is: find $z \in H_{*}^{1}, t>0$ such that:

$\left(\partial_{t} z, v\right)+\left(\partial_{t} \partial_{x} z, \partial_{x} v\right)+\nu\left(\partial_{x} z, \partial_{x} v\right)+\left(u^{\infty} \partial_{x} z+z \partial_{x} u^{\infty}, v\right)=(F, v), \quad \forall v \in H_{*}^{1}$.

In order to obtain the estimation results, we need the following assumptions: (A2): $u_{0} \in H^{2} \cap H_{*}^{1}, \quad f^{\infty} \in L^{2}, \quad f \in L^{\infty}\left((0, \infty), L^{2}\right), \quad\left\|e^{a_{1} t} F(t)\right\|_{0}^{2} \leq M, M>$ 0 .

For the original equation (1)-(3), we have the following stability results. Theorem 2.1: Under the assumption of A2, the solution of equation (1)-(3) satisfies the following regularity estimation

$$
\|u\|_{0}^{2}+\left\|\partial_{x} u\right\|_{0}^{2} \leq\left\|u_{0}\right\|_{0}^{2}+\left\|\partial_{x} u_{0}\right\|_{0}^{2}+C \int_{0}^{t}\|f(s)\|_{-1}^{2} d s .
$$

Proof: Taking the inner product with $u$, we have

$$
\frac{1}{2} \frac{d}{d t}\|u\|_{0}^{2}+\frac{1}{2} \frac{d}{d t}\left\|\partial_{x} u\right\|_{0}^{2}+\nu\left\|\partial_{x} u\right\|_{0}^{2}=(f, u) \leq C\|f\|_{-1}^{2}+\nu\left\|\partial_{x} u\right\|^{2} .
$$

Then

$$
\frac{d}{d t}\left(\|u\|_{0}^{2}+\left\|\partial_{x} u\right\|_{0}^{2}\right) \leq C\|f\|_{-1}^{2} .
$$

That is (16).

Theorem 2.2 Assuming A1 and A2 hold, for $0<a \leq \frac{\nu \lambda_{1}}{4\left(\lambda_{1}+1\right)}, \delta \in(0, a), a_{1}=$ $a-\delta$, we have

$$
\left\|e^{a_{1} t} z\right\|_{0}^{2}+\left\|\partial_{x} e^{a_{1} t} z\right\|_{0}^{2} \leq C\left(\left\|z_{0}\right\|_{1}^{2}, \nu, \lambda_{1}, \delta, M\right) .
$$

Proof: Let $v=e^{2 a t} z$ in(12), we have

$$
\begin{aligned}
\frac{1}{2} \frac{d}{d t}\left(\left\|e^{a t} z\right\|_{0}^{2}+\left\|e^{a t} \partial_{x} z\right\|_{0}^{2}\right) & -a\left(\left\|e^{a t} z\right\|_{0}^{2}+\left\|e^{a t} \partial_{x} z\right\|_{0}^{2}\right) \\
& +\nu\left\|e^{a t} \partial_{x} z\right\|_{0}^{2}+\left(u^{\infty} \partial_{x} e^{a t} z+e^{a t} z \partial_{x} u^{\infty}, e^{a t} z\right)=\left(e^{a t} F, e^{a t} z\right) .
\end{aligned}
$$

From (8),Young's and Poincaréinequality, we have

$$
\begin{aligned}
\frac{d}{d t}\left(\left\|e^{a t} z\right\|_{0}^{2}+\left\|e^{a t} \partial_{x} z\right\|_{0}^{2}\right) & -2 a\left(\left\|e^{a t} z\right\|_{0}^{2}+\left\|e^{a t} \partial_{x} z\right\|_{0}^{2}\right)+\nu\left\|e^{a t} \partial_{x} z\right\|_{0}^{2} \\
\leq & \frac{2}{\sqrt{\lambda_{1}}}\left\|e^{a t} F\right\|_{0}\left\|e^{a t} z_{x}\right\|_{0} \leq \frac{2}{\nu \lambda_{1}}\left\|e^{a t} F\right\|_{0}^{2}+\frac{\nu}{2}\left\|e^{a t} \partial_{x} z\right\|_{0}^{2} .
\end{aligned}
$$

Using Poincaréinequality again

$$
\frac{d}{d t}\left(\left\|e^{a t} z\right\|_{0}^{2}+\left\|e^{a t} \partial_{x} z\right\|_{0}^{2}\right)+\left(\frac{\nu}{2}-2 a\left(\frac{1}{\lambda_{1}}+1\right)\right)\left\|e^{a t} \partial_{x} z\right\|_{0}^{2} \leq \frac{2}{\nu \lambda_{1}}\left\|e^{a t} F\right\|_{0}^{2} .
$$


Integrating $t$ on both sides

$$
e^{2 a t}\left(\|z\|_{0}^{2}+\left\|\partial_{x} z\right\|_{0}^{2}\right) \leq\left\|z_{0}\right\|_{0}^{2}+\left\|\partial_{x} z_{0}\right\|_{0}^{2}+\frac{2}{\nu \lambda_{1}} \int_{0}^{t}\left\|e^{a s} F(s)\right\|_{0}^{2} d s .
$$

multiplying by $e^{-2 \delta t}$, noticing $a=a_{1}+\delta$, then

$$
e^{2 a_{1} t}\left(\|z\|_{0}^{2}+\left\|\partial_{x} z\right\|_{0}^{2}\right) \leq e^{-2 \delta t}\left(\left\|z_{0}\right\|_{0}^{2}+\left\|\partial_{x} z_{0}\right\|_{0}^{2}\right)+\frac{M}{\nu \lambda_{1} \delta}\left(1-e^{-2 \delta t}\right)
$$

Theorem 2.3 Under the assumption of A1 and A2, for $0<a \leq \frac{\nu \lambda_{1}}{4\left(\lambda_{1}+1\right)}$, we have

$$
\left\|\partial_{x} e^{a_{1} t} z\right\|_{0}^{2}+\left\|\partial_{x}^{2} e^{a_{1} t} z\right\|_{0}^{2} \leq C\left(\left\|z_{0}\right\|_{1}^{2},\left\|f^{\infty}\right\|_{-1}, \nu, \lambda_{1}, \delta, M\right)
$$

Proof: Let $v=-e^{2 a t} \partial_{x}^{2} z$ in equation (12)

$$
\begin{aligned}
& \frac{1}{2} \frac{d}{d t}\left(\left\|e^{a t} \partial_{x}^{2} z\right\|_{0}^{2}+\left\|e^{a t} \partial_{x}^{2} z\right\|_{0}^{2}\right)-a\left(\left\|e^{a t} \partial_{x}^{2} z\right\|_{0}^{2}+\left\|e^{a t} \partial_{x}^{2} z\right\|_{0}^{2}\right)+\nu\left\|e^{a t} \partial_{x}^{2} z\right\|_{0}^{2} \\
= & \left(e^{a t} F,-\partial_{x}^{2} e^{a t} z\right)+\left(z e^{a t} \partial_{x} z, e^{a t} \partial_{x}^{2} z\right)+\left(u^{\infty} \partial_{x} e^{a t} z+e^{a t} z \partial_{x} u^{\infty}, \partial_{x}^{2} e^{a t} z\right) .
\end{aligned}
$$

For the first term at the right end, it can be obtained from Young' s inequality:

$$
\left(e^{a t} F,-\partial_{x}^{2} e^{a t} z\right) \leq \frac{1}{\nu}\left\|e^{a t} F\right\|_{0}^{2}+\frac{\nu}{4}\left\|e^{a t} \partial_{x}^{2} z\right\|_{0}^{2}
$$

For the second term at the right end, it can be obtained from Young' s inequality and interpolation inequality

$$
\begin{aligned}
\left(z e^{a t} \partial_{x} z, e^{a t} \partial_{x}^{2} z\right) \leq\|z\|_{0}\left\|e^{a t} \partial_{x} z\right\|_{L^{\infty}}\left\|e^{a t} \partial_{x}^{2} z\right\|_{0} & \leq C\|z\|_{0}\left\|e^{a t} \partial_{x} z\right\|_{0}^{\frac{1}{2}}\left\|e^{a t} \partial_{x}^{2} z\right\|_{0}^{\frac{1}{2}}\left\|e^{a t} \partial_{x}^{2} z\right\|_{0} \\
& \leq C(\nu)\|z\|_{0}^{4}\left\|e^{a t} \partial_{x} z\right\|_{0}^{2}+\frac{\nu}{4}\left\|e^{a t} \partial_{x}^{2} z\right\|_{0}^{2}
\end{aligned}
$$

For the third term at the right end, it can be obtained from Young's inequality, Poincaré inequality and interpolation inequality:

$$
\begin{aligned}
\left(u^{\infty} \partial_{x} e^{a t} z\right. & \left.+e^{a t} z \partial_{x} u^{\infty}, \partial_{x}^{2} e^{a t} z\right) \\
& \leq\left(C(\nu)\left\|u^{\infty}\right\|_{L^{\infty}}^{2}+C(\nu) \frac{1}{\lambda_{1}}\left\|\partial_{x} u^{\infty}\right\|_{L^{\infty}}^{2}\right)\left\|e^{a t} \partial_{x} z\right\|_{0}^{2}+\frac{\nu}{4}\left\|e^{a t} \partial_{x}^{2} z\right\|_{0}^{2} \\
& \leq C\left(\nu, \lambda_{1},\left\|f^{\infty}\right\|_{-1}\right)\left\|e^{a t} \partial_{x} z\right\|_{0}^{2}+\frac{\nu}{4}\left\|e^{a t} \partial_{x}^{2} z\right\|_{0}^{2} .
\end{aligned}
$$

Then

$$
\begin{aligned}
& \frac{d}{d t}\left(\left\|e^{a t} \partial_{x} z\right\|_{0}^{2}+\left\|e^{a t} \partial_{x}^{2} z\right\|_{0}^{2}\right)-2 a\left(\left\|e^{a t} \partial_{x} z\right\|_{0}^{2}+\left\|e^{a t} \partial_{x}^{2} z\right\|_{0}^{2}\right)+\frac{\nu}{2}\left\|e^{a t} \partial_{x}^{2} z\right\|_{0}^{2} \\
\leq & \frac{2}{\nu}\left\|e^{a t} F\right\|_{0}^{2}+C(\nu)\|z\|_{0}^{4}\left\|e^{a t} \partial_{x} z\right\|_{0}^{2}+C\left(\nu, \lambda_{1},\left\|f^{\infty}\right\|_{-1}\right)\left\|e^{a t} \partial_{x} z\right\|_{0}^{2} .
\end{aligned}
$$

From Poincaré inequality, we obtain

$$
\begin{aligned}
& \frac{d}{d t}\left(\left\|\partial_{x} e^{a t} z\right\|_{0}^{2}+\left\|\partial_{x}^{2} e^{a t} z\right\|_{0}^{2}\right)+\left(\frac{\nu}{2}-2 a\left(\frac{1}{\lambda_{1}}+1\right)\right)\left\|e^{a t} \partial_{x}^{2} z\right\|_{0}^{2} \\
\leq & \frac{2}{\nu}\left\|e^{a t} F\right\|_{0}^{2}+C(\nu)\|z\|_{0}^{4}\left\|e^{a t} \partial_{x} z\right\|_{0}^{2}+C\left(\nu, \lambda_{1},\left\|f^{\infty}\right\|_{-1}\right)\left\|e^{a t} \partial_{x} z\right\|_{0}^{2} .
\end{aligned}
$$


Integrating $t$ on the both sides and multiplying by $e^{-2 \delta t}$, that is

$$
\begin{aligned}
\left\|\partial_{x} e^{a_{1} t} z\right\|_{0}^{2}+\left\|\partial_{x}^{2} e^{a_{1} t} z\right\|_{0}^{2} \leq & e^{-2 \delta t}\left(\left\|z_{0}\right\|_{0}^{2}+\left\|\partial_{x} z_{0}\right\|_{0}^{2}\right)+\frac{2}{\nu} e^{-2 \delta t} \int_{0}^{t}\left\|e^{a s} F(s)\right\|_{0}^{2} d s \\
& +C(\nu) e^{-2 \delta t} \int_{0}^{t}\|z\|_{0}^{4}\left\|e^{a s} \partial_{x} z\right\|_{0}^{2} d s \\
& +C\left(\nu, \lambda_{1},\left\|f^{\infty}\right\|_{-1}\right) e^{-2 \delta t} \int_{0}^{t}\left\|e^{a s} \partial_{x} z\right\|_{0}^{2} d s \\
\leq & e^{-2 \delta t}\left(\left\|z_{0}\right\|_{0}^{2}+\left\|\partial_{x} z_{0}\right\|_{0}^{2}\right)+\frac{M}{\nu \delta}\left(1-e^{-2 \delta t}\right) \\
& +e^{-4 a_{1} t}\left(1-e^{-2 \delta t}\right) C\left(\left\|z_{0}\right\|_{1}^{2}, \nu, \lambda_{1}, \delta, M\right) \\
& +\left(1-e^{-2 \delta t}\right) C\left(\left\|z_{0}\right\|_{1}^{2}, \nu,\left\|f^{\infty}\right\|_{-1}, \lambda_{1}, \delta, M\right)
\end{aligned}
$$

We consider the following assumptions (A3): $u_{0} \in H^{2} \cap H_{*}^{1}, \quad f^{\infty} \in$ $L^{2}, \quad f \in L^{\infty}\left((0, \infty), L^{2}\right), \sup _{t>0}\|f(t)\|_{0}^{2} \leq M_{1}, \lim _{t \rightarrow \infty}\|F(t)\|_{0}=0$.

\section{Theorem 2.4}

Under the assumption that $\mathrm{A} 1$ and $\mathrm{A} 3$, for a given $\varepsilon>0$, there is a $T>$ 0 , such that for all $t \geq T,\|F(t)\|_{0}<\varepsilon$ and the following estimates holds

$$
\|z\|_{0}^{2}+\left\|\partial_{x} z\right\|_{0}^{2}+\left\|\partial_{x}^{2} z\right\|_{0}^{2} \leq C e^{-2 a(t-T)}+C \varepsilon^{2},
$$

where $C=C\left(\left\|z_{0}\right\|_{1}^{2}, a, \nu,\left\|f^{\infty}\right\|_{-1}, \lambda_{1}, \delta, M_{1}\right)$.

Proof: From (18), we have

$$
\left(\|z\|_{0}^{2}+\left\|\partial_{x} z\right\|_{0}^{2}\right) \leq e^{-2 a t}\left(\left\|z_{0}\right\|_{0}^{2}+\left\|\partial_{x} z_{0}\right\|_{0}^{2}\right)+\frac{2}{\nu \lambda_{1}} e^{-2 a t} \int_{0}^{t}\left\|e^{a s} F(s)\right\|_{0}^{2} d s .
$$

From $\lim _{t \rightarrow \infty}\|F(t)\|_{0}=0$, for all $\varepsilon>0$, there is a $T>0$, such that $t \geq T$, for $\|F(t)\|_{0} \leq \varepsilon$. So we split the right integral term into two parts, $(0, T)$ 和 $(T, t)$, so we have

$$
\left(\|z\|_{0}^{2}+\left\|\partial_{x} z\right\|_{0}^{2}\right) \leq C\left(\nu, a, \lambda_{1}\right) e^{-2 a(t-T)}\left(\left\|z_{0}\right\|_{0}^{2}+\left\|\partial_{x} z_{0}\right\|_{0}^{2}+M_{1}\right)+C \varepsilon^{2} .
$$

For the (20) equation, integrate on both sides and multiple by $e^{-2 a t}$, there are

$$
\begin{aligned}
\left\|\partial_{x} z\right\|_{0}^{2}+\left\|\partial_{x}^{2} z\right\|_{0}^{2} \leq & e^{-2 a t}\left(\left\|z_{0}\right\|_{0}^{2}+\left\|\partial_{x} z_{0}\right\|_{0}^{2}\right)+\frac{2}{\nu} e^{-2 a t} \int_{0}^{t}\left\|e^{a s} F(s)\right\|_{0}^{2} d s \\
& +C(\nu) e^{-2 a t} \int_{0}^{t}\|z\|_{0}^{4}\left\|e^{a s} \partial_{x} z\right\|_{0}^{2} d s+C\left(\nu, \lambda_{1},\left\|f^{\infty}\right\|_{-1}\right) e^{-2 a t} \int_{0}^{t}\left\|e^{a s} \partial_{x} z\right\|_{0}^{2} d s .
\end{aligned}
$$

At the same time

$$
\left\|\partial_{x} z\right\|_{0}^{2}+\left\|\partial_{x}^{2} z\right\|_{0}^{2} \leq C e^{-2 a(t-T)}\left(\left\|\partial_{x} z_{0}\right\|_{0}^{2}+\left\|\partial_{x}^{2} z_{0}\right\|_{0}^{2}\right)+C \varepsilon^{2} .
$$

Then we obtain (21).

\section{$\S 3 \quad$ Stability analysis of numerical schemes}

In this section, we will give a time semi-discrete scheme and analyze the unconditional stability of this time discrete scheme. Given a positive integer $M$, 
let $t_{n}=n \Delta t, n=0,1, \ldots, M$, where $\Delta t=T / M$ is the time step size, $T$ be the time.

\section{C-N scheme.}

First step $n=0$ :

$$
\frac{u^{1}-u^{0}}{\Delta t}+\partial_{x} u^{1}-\frac{\partial_{x}^{2} u^{1}-\partial_{x}^{2} u^{0}}{\Delta t}+\frac{1}{3}\left(2 u^{0} \partial_{x} u^{1}+u^{1} \partial_{x} u^{0}\right)-\nu \partial_{x}^{2} u^{1}=0 .
$$

When $n>1$, we have

$$
\begin{aligned}
& \frac{u^{n+1}-u^{n}}{\Delta t}+\partial_{x} u^{n+\frac{1}{2}}-\frac{\partial_{x}^{2} u^{n+1}-\partial_{x}^{2} u^{n}}{\Delta t}-\nu \partial_{x}^{2} u^{n+\frac{1}{2}} \\
& +\frac{1}{6}\left(2 \partial_{x} u^{n+\frac{1}{2}}\left(3 u^{n}-u^{n-1}\right)+u^{n+\frac{1}{2}} \partial_{x}\left(3 u^{n}-u^{n-1}\right)\right)=0,
\end{aligned}
$$

where

$$
u^{n+\frac{1}{2}}=\frac{u^{n+1}+u^{n}}{2} .
$$

Theorem 3.1 The solution of equation (22)-(23) satisfies the following estimation:

$$
E\left(u^{n+1}\right) \leq E\left(u^{0}\right), \quad n=0,1, \cdots, M-1,
$$

here

$$
E\left(u^{n}\right)=\left\|u^{n}\right\|_{0}^{2}+\left\|\partial_{x} u^{n}\right\|_{0}^{2} .
$$

Proof: Taking the inner product with $2 \Delta t u^{1}$ in (22). Note that

$\left(2 \partial_{x} u^{1} u^{0}+u^{1} \partial_{x} u^{0}, u^{1}\right)=\left(\partial_{x} u^{1} u^{0}+\partial_{x}\left(u^{1} u^{0}\right), u^{1}\right)=\left(\partial_{x} u^{1} u^{0}, u^{1}\right)-\left(u^{0} u^{1}, \partial_{x} u^{1}\right)=0$.

Then

$\left\|u^{1}\right\|_{0}^{2}-\left\|u^{0}\right\|_{0}^{2}+\left\|u^{1}-u^{0}\right\|_{0}^{2}+\left\|\partial_{x} u^{1}\right\|_{0}^{2}-\left\|\partial_{x} u^{0}\right\|_{0}^{2}+\left\|\partial_{x} u^{1}-\partial_{x} u^{0}\right\|_{0}^{2}+2 \Delta t \nu\left\|\partial_{x} u^{1}\right\|_{0}^{2}=0$.

Then we obtain the first step of proof. Taking the inner product with $2 \Delta t u^{n+\frac{1}{2}}$ in (23), we have

$$
\begin{aligned}
& \left(2 \partial_{x} u^{n+\frac{1}{2}}\left(3 u^{n}-u^{n-1}\right)+u^{n+\frac{1}{2}} \partial_{x}\left(3 u^{n}-u^{n-1}\right), u^{n+\frac{1}{2}}\right) \\
= & \left(\partial_{x} u^{n+\frac{1}{2}}\left(3 u^{n}-u^{n-1}\right), u^{n+\frac{1}{2}}\right)+\left(\partial_{x}\left(u^{n+\frac{1}{2}}\left(3 u^{n}-u^{n-1}\right)\right), u^{n+\frac{1}{2}}\right) \\
= & \left(\partial_{x} u^{n+\frac{1}{2}}\left(3 u^{n}-u^{n-1}\right), u^{n+\frac{1}{2}}\right)-\left(\left(3 u^{n}-u^{n-1}\right) u^{n+\frac{1}{2}}, \partial_{x} u^{n+\frac{1}{2}}\right) \\
= & 0 .
\end{aligned}
$$

Then we have

$$
\left\|u^{n+1}\right\|_{0}^{2}-\left\|u^{n}\right\|_{0}^{2}+\left\|\partial_{x} u^{n+1}\right\|_{0}^{2}-\left\|\partial_{x} u^{n}\right\|_{0}^{2}+2 \Delta t \alpha\left\|\partial_{x} u^{n+\frac{1}{2}}\right\|_{0}^{2}=0 .
$$

We get proof of the theorem. 


\section{$\S 4$ Error estimation for full discrete scheme}

Here we will study the spatial discretization method of the scheme (22)$(23)$, let $S_{N}=\operatorname{span}\{\exp (-i k x):|k| \leq N\}$, and denote projection $\pi_{N}: L^{2}(\Lambda) \rightarrow$ $S_{N}$, that is

$$
\left(\pi_{N} v-v, \psi\right)=0, \forall \psi \in S_{N},
$$

and $H^{1}$ projection $\pi_{N}^{1}: H^{1}(\Lambda) \rightarrow S_{N}$, that is

$$
\left(\partial_{x}\left(\pi_{N}^{1} v-v\right), \partial_{x} \psi\right)=0, \quad\left(\pi_{N}^{1} v-v, \psi\right)=0, \quad \forall \psi \in S_{N}
$$

From [15]we have

$$
\begin{aligned}
& \left\|u-\pi_{N} u\right\|_{0} \leq N^{-m}\|u\|_{m}, \quad \forall u \in H^{m}(\Lambda), m>0, \\
& \left\|u-\pi_{N}^{1} u\right\|_{k} \leq N^{k-m}\|u\|_{m}, \quad \forall u \in H^{m}(\Lambda), m>0, k=0,1 .
\end{aligned}
$$

C-N/F-G. The fully discrete scheme of equation (1) is: find $u_{N}^{n+1} \in S_{N}$, such that:

when $n=0$

$$
\begin{aligned}
& \frac{1}{\Delta t}\left(u_{N}^{1}-u_{N}^{0}, \psi_{N}\right)+\left(\partial_{x} u_{N}^{1}, \psi_{N}\right)+\frac{1}{\Delta t}\left(\partial_{x} u_{N}^{1}-\partial_{x} u_{N}^{0}, \partial_{x} \psi_{N}\right) \\
& +\frac{1}{3}\left(2 u_{N}^{0} \partial_{x} u_{N}^{1}+u^{1} \partial_{x} u_{N}^{0}, \psi_{N}\right)+\nu\left(\partial_{x} u^{1}, \partial_{x} \psi_{N}\right)=0, n \geq 0, \forall \psi_{N} \in S_{N} .
\end{aligned}
$$

$n \geq 1$

$$
\begin{aligned}
& \frac{1}{\Delta t}\left(u_{N}^{n+1}-u_{N}^{n}, \psi_{N}\right)+\left(\partial_{x} u_{N}^{n+\frac{1}{2}}, \psi_{N}\right)+\frac{1}{\Delta t}\left(\partial_{x} u_{N}^{n+1}-\partial_{x} u_{N}^{n}, \partial_{x} \psi_{N}\right)+\nu\left(\partial_{x} u_{N}^{n+\frac{1}{2}}, \partial_{x} \psi_{N}\right) \\
& +\frac{1}{6}\left(2 \partial_{x} u_{N}^{n+\frac{1}{2}}\left(3 u_{N}^{n}-u_{N}^{n-1}\right)+u_{N}^{n+\frac{1}{2}} \partial_{x}\left(3 u_{N}^{n}-u_{N}^{n-1}\right), \psi_{N}\right)=0, n \geq 1, \forall \psi_{N} \in S_{N} .
\end{aligned}
$$

Denote

$\widetilde{e}_{N}^{n}=\pi_{N}^{1} u\left(\cdot, t_{n}\right)-u_{N}^{n}, \quad \widehat{e}_{N}^{n}=u\left(\cdot, t_{n}\right)-\pi_{N}^{1} u\left(\cdot, t_{n}\right), \quad e_{N}^{n}=u\left(\cdot, t_{n}\right)-u_{N}^{n}=\widetilde{e}_{N}^{n}+\widehat{e}_{N}^{n}, n \geq 0$,

and error function $R^{n}(x)=r_{1}^{n}(x)+r_{2}^{n}(x)$, where

$$
\begin{aligned}
r_{1}^{n}(x) & :=\frac{u\left(x, t_{n+1}\right)-u\left(x, t_{n}\right)}{\Delta t}-\partial_{t} u\left(x, t_{n+\frac{1}{2}}\right), \\
r_{2}^{n}(x) & :=\frac{\partial_{x}^{2} u\left(x, t_{n+1}\right)-\partial_{x}^{2} u\left(x, t_{n}\right)}{\Delta t}-\partial_{x}^{2} \partial_{t} u\left(x, t_{n+\frac{1}{2}}\right) .
\end{aligned}
$$

That is

$$
\left\|r_{1}^{n+1}\right\|_{0}^{2} \leq c \Delta t^{4}, \quad\left\|r_{2}^{n+1}\right\|_{0}^{2} \leq c \Delta t^{4}, \quad\left\|R^{n}\right\|_{0}^{2} \leq c \Delta t^{4} .
$$

We have the following stability results.

Theorem 4.1 If $\left\{u_{N}^{n+1}\right\}$ is a solution of the full discrete scheme (27)-(28), then we have

$$
E\left(u_{N}^{n+1}\right) \leq E\left(u_{N}^{0}\right), \quad n=0,1, \ldots, M-1 .
$$


Theorem 4.2 The solution of the full discrete scheme (27)-(28) satisfies the following error estimate:

$$
\left\|u\left(\cdot, t_{k}\right)-u_{N}^{k}\right\|_{1} \leq c\left(\Delta t^{2}+N^{1-m}\right), k=0,1,2, \ldots, M .
$$

Proof: When $f=0$, from (1) and (28) we have

$$
\begin{aligned}
& \left.\left(\frac{\widetilde{e}_{N}^{n+1}-\widetilde{e}_{N}^{n}}{\Delta t}, \psi_{N}\right)+\left(\partial_{x} \widetilde{e}_{N}^{n+\frac{1}{2}}, \psi_{N}\right)+\frac{1}{\Delta t}\left(\partial_{x} \widetilde{e}_{N}^{n+1}-\partial_{x} \widetilde{e}_{N}^{n}\right), \partial_{x} \psi_{N}\right)+\nu\left(\partial_{x} \widetilde{e}_{N}^{n+\frac{1}{2}}, \partial_{x} \psi_{N}\right) \\
& +\left(u\left(\cdot, t_{n+\frac{1}{2}}\right) \partial_{x} u\left(\cdot, t_{n+\frac{1}{2}}\right)-\frac{1}{6}\left(2 \partial_{x} u_{N}^{n+\frac{1}{2}}\left(3 u_{N}^{n}-u_{N}^{n-1}\right)+u_{N}^{n+\frac{1}{2}} \partial_{x}\left(3 u_{N}^{n}-u_{N}^{n-1}\right), \psi_{N}\right)\right. \\
= & \left(R^{n}, \psi_{N}\right)+\left(\left(\pi_{N}^{1}-I\right) \partial_{x} u\left(\cdot, t_{n+\frac{1}{2}}\right), \psi_{N}\right)+\frac{1}{\Delta t}\left(\left(\pi_{N}^{1}-I\right)\left(u\left(\cdot, t_{n+1}\right)-u\left(\cdot, t_{n}\right)\right), \psi_{N}\right) \\
& +\frac{1}{\Delta t}\left(\left(\pi_{N}^{1}-I\right)\left(\partial_{x}^{2} u\left(\cdot, t_{n+1}\right)-\partial_{x}^{2} u\left(\cdot, t_{n}\right)\right), \psi_{N}\right) .
\end{aligned}
$$

$\operatorname{Let} \psi_{N}=2 \Delta t \tilde{e}_{N}^{n+\frac{1}{2}}$, we obtain

$$
\begin{aligned}
& E\left(\widetilde{e}^{n+1}\right)-E\left(\widetilde{e}^{n}\right) \\
\leq & 2 \Delta t\left\|R^{n+1}\right\|_{0}\left\|\widetilde{e}_{N}^{n+\frac{1}{2}}\right\|_{0}+2 \Delta t\left\|\left(\pi_{N}^{1}-I\right) \partial_{x} u\left(\cdot, t_{n+\frac{1}{2}}\right)\right\|_{0}\left\|\widetilde{e}_{N}^{n+\frac{1}{2}}\right\|_{0} \\
& +2\left\|\left(\pi_{N}^{1}-I\right)\left(u\left(\cdot, t_{n+1}\right)-u\left(\cdot, t_{n}\right)\right)\right\|_{0}\left\|\widetilde{e}_{N}^{n+\frac{1}{2}}\right\|_{0} \\
& +2\left\|\left(\pi_{N}^{1}-I\right)\left(\partial_{x} u\left(\cdot, t_{n+1}\right)-\partial_{x} u\left(\cdot, t_{n}\right)\right)\right\|_{0}\left\|\partial_{x} \widetilde{e}_{N}^{n+\frac{1}{2}}\right\|_{0} \\
& +2 \Delta t\left\|u\left(\cdot, t_{n+\frac{1}{2}}\right) \partial_{x} u\left(\cdot, t_{n+\frac{1}{2}}\right)-\frac{1}{6}\left(2 \partial_{x} u_{N}^{n+1}\left(3 u_{N}^{n}-u_{N}^{n-1}\right)+u_{N}^{n+1} \partial_{x}\left(3 u_{N}^{n}-u_{N}^{n-1}\right)\right)\right\|_{0}\left\|\widetilde{e}_{N}^{n+1}\right\|_{0}
\end{aligned}
$$

Notice that

$$
u\left(\cdot, t_{n+\frac{1}{2}}\right) \partial_{x} u\left(\cdot, t_{n+\frac{1}{2}}\right)-\frac{1}{6}\left(2 \partial_{x} u_{N}^{n+\frac{1}{2}}\left(3 u_{N}^{n}-u_{N}^{n-1}\right)+u_{N}^{n+\frac{1}{2}} \partial_{x}\left(3 u_{N}^{n}-u_{N}^{n-1}\right)=D_{1}+D_{2},\right.
$$

where

$$
\begin{aligned}
D_{1}= & \frac{2}{3} u\left(\cdot, t_{n+\frac{1}{2}}\right) \partial_{x} u\left(\cdot, t_{n+\frac{1}{2}}\right)-\frac{1}{3}\left(3 u_{N}^{n}-u_{N}^{n-1}\right) \partial_{x} u_{N}^{n+\frac{1}{2}} \\
= & \frac{2}{3} u\left(\cdot, t_{n+\frac{1}{2}}\right) \partial_{x} u\left(\cdot, t_{n+\frac{1}{2}}\right)-\frac{1}{3}\left(3 u\left(\cdot, t_{n}\right)-u\left(\cdot, t_{n-1}\right)\right) \partial_{x} u\left(\cdot, t_{n+\frac{1}{2}}\right) \\
& +\frac{1}{3}\left(3 u\left(\cdot, t_{n}\right)-u\left(\cdot, t_{n-1}\right)\right) \partial_{x} u\left(\cdot, t_{n+\frac{1}{2}}\right)-\frac{1}{3}\left(3 u_{N}^{n}-u_{N}^{n-1}\right) \partial_{x} u\left(\cdot, t_{n+\frac{1}{2}}\right) \\
& +\frac{1}{3}\left(3 u_{N}^{n}-u_{N}^{n-1}\right) \partial_{x} u\left(\cdot, t_{n+\frac{1}{2}}\right)-\frac{1}{3}\left(3 u_{N}^{n}-u_{N}^{n-1}\right) \partial_{x} u_{N}^{n+\frac{1}{2}}, \\
D_{2}= & \frac{1}{3} u\left(t_{n+\frac{1}{2}}\right) \partial_{x} u\left(t_{n+\frac{1}{2}}\right)-\frac{1}{6} u_{N}^{n+\frac{1}{2}} \partial_{x}\left(3 u_{N}^{n}-u_{N}^{n-1}\right) \\
= & \frac{1}{3} u\left(t_{n+\frac{1}{2}}\right) \partial_{x} u\left(\cdot, t_{n+1}\right)-\frac{1}{6} u\left(\cdot, t_{n+\frac{1}{2}}\right) \partial_{x}\left(3 u\left(\cdot, t_{n}\right)-u\left(\cdot, t_{n-1}\right)\right) \\
& +\frac{1}{6} u\left(\cdot, t_{n+\frac{1}{2}}\right) \partial_{x}\left(3 u\left(\cdot, t_{n}\right)-u\left(\cdot, t_{n-1}\right)\right)-\frac{1}{6} u_{N}^{n+\frac{1}{2}} \partial_{x}\left(3 u\left(\cdot, t_{n}\right)-u\left(\cdot, t_{n-1}\right)\right) \\
& +\frac{1}{6} u_{N}^{n+\frac{1}{2}} \partial_{x}\left(3 u\left(\cdot, t_{n}\right)-u\left(\cdot, t_{n-1}\right)\right)-\frac{1}{6} u_{N}^{n+\frac{1}{2}} \partial_{x}\left(3 u_{N}^{n}-u_{N}^{n-1}\right) .
\end{aligned}
$$


From taylor expansion and Young' s inequality, we derive

$$
\begin{aligned}
\frac{1}{3}\left\|D_{1}\right\|_{0}^{2} \leq & \Delta t^{3}\left\|\partial_{x} u\left(\cdot, t_{n+\frac{1}{2}}\right)\right\|_{\infty}^{2} \int_{t_{n}}^{t_{n+1}}\left\|\partial_{t}^{2} u(\cdot, t)\right\|_{0}^{2} d t \\
& +\left\|\partial_{x} u\left(\cdot, t_{n+\frac{1}{2}}\right)\right\|_{\infty}^{2}\left\|3 e_{N}^{n}-e_{N}^{n-1}\right\|_{0}^{2}+\left\|3 u_{N}^{n}-u_{N}^{n-1}\right\|_{\infty}^{2}\left\|\partial_{x} e_{N}^{n+\frac{1}{2}}\right\|_{0}^{2} \\
\leq & c\left(\Delta t^{4}+\left\|3 e_{N}^{n}-e_{N}^{n-1}\right\|_{0}^{2}+\left\|\partial_{x} e_{N}^{n+\frac{1}{2}}\right\|_{0}^{2}\right) . \\
\frac{1}{3}\left\|D_{2}\right\|_{0}^{2} \leq & \Delta t^{3}\left\|u\left(\cdot, t_{n+\frac{1}{2}}\right)\right\|_{\infty}^{2} \int_{t_{n}}^{t_{n+1}}\left\|\partial_{x} \partial_{t}^{2} u(\cdot, t)\right\|_{0}^{2} d t \\
& +\left\|\partial_{x}\left(3 u\left(\cdot, t_{n}\right)-u\left(\cdot, t_{n-1}\right)\right)\right\|_{\infty}^{2}\left\|e_{N}^{n+\frac{1}{2}}\right\|_{0}^{2}+\left\|u_{N}^{n+\frac{1}{2}}\right\|_{\infty}^{2}\left\|\partial_{x}\left(3 e_{N}^{n}-e_{N}^{n-1}\right)\right\|_{0}^{2} \\
\leq & c\left(\Delta t^{4}+\left\|\partial_{x}\left(3 e_{N}^{n}-e_{N}^{n-1}\right)\right\|_{0}^{2}+\left\|e_{N}^{n+\frac{1}{2}}\right\|_{0}^{2}\right)
\end{aligned}
$$

Using Cauchy-Schwarz inequality

$$
\begin{aligned}
E\left(\widetilde{e}^{n+1}\right)-E\left(\widetilde{e}^{n}\right) \leq & \Delta t\left\|R^{n}\right\|_{0}^{2}+\Delta t\left\|\left(\pi_{N}^{1}-I\right) \partial_{x} u\left(\cdot, t_{n+\frac{1}{2}}\right)\right\|_{0}^{2}+2 \Delta t\left\|\widetilde{e}_{N}^{n+1}\right\|_{0}^{2} \\
& +\int_{t_{n}}^{t_{n+1}}\left\|\left(\pi_{N}^{1}-I\right) \partial_{t} u(\cdot, t)\right\|_{0}^{2} d t+\Delta t\left\|\widetilde{e}_{N}^{n+1}\right\|_{0}^{2}, \\
& +\int_{t_{n}}^{t_{n+1}}\left\|\left(\pi_{N}^{1}-I\right) \partial_{x} \partial_{t} u(\cdot, t)\right\|_{0}^{2} d t+\Delta t\left\|\partial_{x} \widetilde{e}_{N}^{n+1}\right\|_{0}^{2}, \\
& +c \Delta t\left(\Delta t^{4}+\left\|3 e_{N}^{n}-e_{N}^{n-1}\right\|_{1}^{2}+\left\|e_{N}^{n+\frac{1}{2}}\right\|_{1}^{2}+\left\|\widetilde{e}_{N}^{n+1}\right\|_{0}\right) .
\end{aligned}
$$

Summing up for $n=1, \ldots, k$, and using (26)-(29), we have

$$
\begin{aligned}
E\left(\widetilde{e}^{k+1}\right)-E\left(\widetilde{e}^{1}\right) \leq & c\left(\Delta t^{2}+N^{1-m}\right) \\
& +c \Delta t \sum_{n=1}^{k}\left(\left\|3 \widetilde{e}_{N}^{n}-\widetilde{e}_{N}^{n-1}\right\|_{1}^{2}+\left\|\widetilde{e}_{N}^{n+1}\right\|_{1}^{2}\right), n=1,2, \ldots, M-1 .
\end{aligned}
$$

For the first step

$$
E\left(\widetilde{e}^{1}\right) \leq c\left(\Delta t^{4}+N^{2-2 m}\right)
$$

Substituting (34)into (33), and using the discrete Gronwall lemma we obtain (31).

\section{$\S 5$ Numerical experiment}

In this section, we will use numerical examples to verify the accuracy of theoretical analysis. First we let $u_{N}^{n}(x)=\sum_{k=-N / 2}^{N / 1-1} \hat{u}_{k}^{n} \exp (-i 2 \pi k x / L)$ in $(27)$ we have:

\section{C-N/F-G scheme:}

$$
\begin{aligned}
& \frac{1}{\Delta t}\left(\hat{u}_{k}^{n+1}-\hat{u}_{k}^{n}\right)\left(1+(2 \pi k / L)^{2}\right)+\left(i 2 \pi k / L+\nu(2 \pi k / L)^{2}\right) \hat{u}_{k}^{n+\frac{1}{2}} \\
& +\frac{1}{6}\left\{2\left(3 u_{N}^{n}-u_{N}^{n-1}\right) \partial_{x} u_{N}^{n+\frac{1}{2}}+\partial_{x}\left(3 u_{N}^{n}-u_{N}^{n-1}\right) u_{N}^{n+\frac{1}{2}}\right\}_{k}=0,
\end{aligned}
$$

$\hat{f}_{k}$ or $\{f\}_{k}$ represent the $k$ Fourier coefficients of $f$. 


\subsection{Validity of numerical solution}

Define the time convergence order:

$$
\text { Rate }:=\log _{2}\left(\frac{\left\|u_{N}^{n, 2 \Delta t}-u_{N}^{2 n, \Delta t}\right\|_{1}}{\left\|u_{N}^{2 n, \Delta t}-u_{N}^{4 n, \Delta t / 2}\right\|_{1}}\right) .
$$

Let $N=60, L=2 \pi, T=1$, and $u(x, 0)=\sin (2 \pi x)$.

It can be clearly seen from Table 5.1 that when $\Delta t \rightarrow 0$, the error order of the format in the time direction is close to 2 order, which is consistent with the proof of the theorem.

Table 5.1 Time Convergence Order.

\begin{tabular}{cccccc}
\hline$\nu \backslash \Delta t$ & $\Delta t=0.1$ & $\Delta t=0.05$ & $\Delta t=0.01$ & $\Delta t=0.005$ & $\Delta t=0.001$ \\
\hline$\nu=0$ & 2.0007 & 2.0004 & 2.0001 & 2.0000 & 2.0000 \\
$\nu=1$ & 2.0540 & 2.0250 & 2.0047 & 2.0023 & 2.0005 \\
\hline
\end{tabular}

\subsection{Asymptotic Attenuation of Solution}

Taking $u(x, t)=\left(1+e^{-t}\right) \sin (x)$ in equation (1). Then $f(x, t)=-2 e^{-t} \sin (x)+$ $\left(1+e^{-t}\right)^{2} \sin (x) \cos (x)+\left(1+e^{-t}\right)(\nu \sin (x)+\cos (x))$, notice that $u^{\infty}(x)=$ $\sin (x), f^{\infty}(x)=\sin (x) \cos (x)+\nu \sin (x)+\cos (x)$. Obviously $f, f-f^{\infty}$ satisfies the hypothesis of A2, A3, so the conclusion of Theorem $2.2-2.4$ holds.

We take $N=60, L=4 \pi, \Delta t=0.01, u_{0}=\sin (x), \nu=1$, as can be seen from fig. 1 , the numerical solution of $z(x, t)$ gradually converges to 0 when $t$ gradually becomes larger.

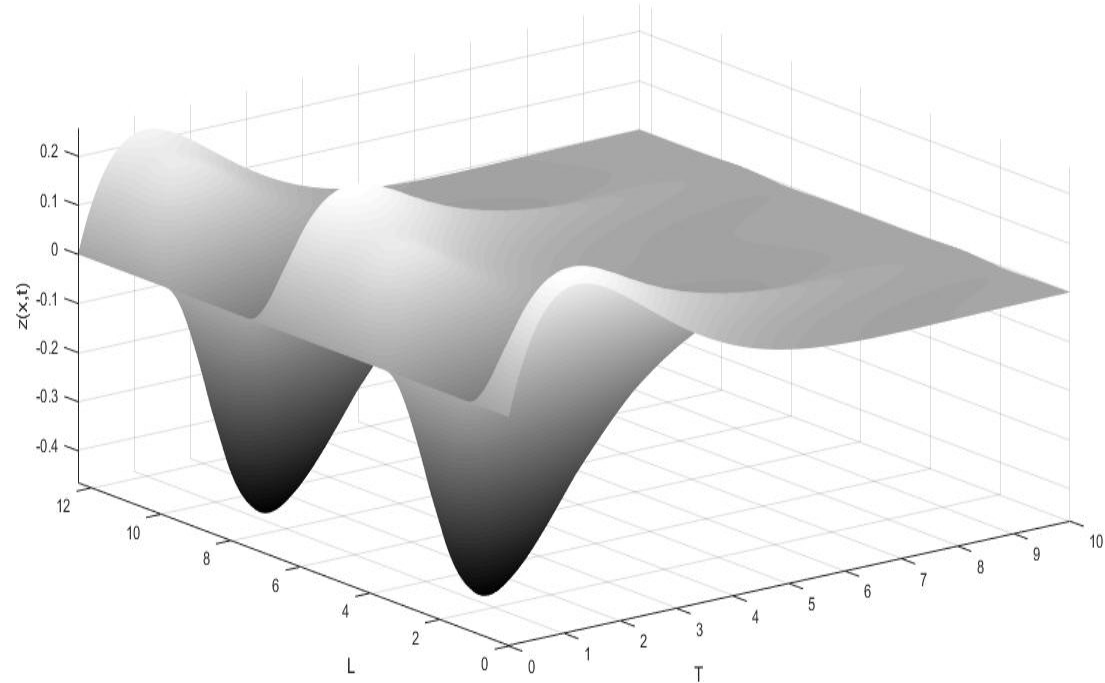

Fig 1. The numerical solution of $z(x, t)$ when $t=10$.

\section{References}

[1] Benjamin, T. B., Bona, J. L., and Mahony, J. J., Model equations for long waves in nonlinear dispersive systems. Philosophical Transactions of the Royal Society of London, 1972, 272(1220):47-78. 
[2] Medeiros, L. A., and Menzala, G. P., Existence and uniqueness for periodic solutions of the benjamin-bona-mahony equation. Siam Journal on Mathematical Analysis, 1977, 8(5):792-799.

[3] Amick, C. J., Bona, J. L., and Schonbek, M. E., Decay of solutions of some nonlinear wave equations. Journal of Differential Equations, 1989, 81(1):1-49, 1989 .

[4] Biler, P., Long time behavior of solutions of the generalized benjamin-bonamahony equation in two space dimensions. Differential and Integral Equations, 1992, 5(4):891-901.

[5] Mei, M., Lq-decay rates of solutions for benjamin-bona-mahony-burgers equations. Journal of Differential Equations, 1999,158(2):314-340.

[6] Chen, M., and Goubet, O., Long-time asymptotic behavior of dissipative boussinesq system. Discrete and Continuous Dynamical Systems-Series A, 2012, 17(3):509-528.

[7] Zhang, L. H., Decay of solution of generalized benjamin-bona-mahony-burgers equations in $n \mathrm{n}$-space dimensions. Nonlinear Analysis Theory Methods and Applications, 1995, 25(12):1343-1369.

[8] Fang, S., and Guo, B., The decay rates of solutions of generalized benjaminbona-mahony equations in multi-dimensions. Nonlinear Analysis Theory Methods and Applications, 2008, 69(7):2230-2235.

[9] Kundu, S., Pani, A. K., and Khebchareon, M., Asymptotic analysis and optimal error estimates for benjamin-bona-mahony-burgers type equations. submitted.

[10] Omrani, K., The convergence of fully discrete galerkin approximations for the benjamin-bona-mahony (bbm) equation. Applied Mathematics and Computation, 2006,180(2):614-621.

[11] Achouri, T., Khiari, N., and Omrani, K., On the convergence of difference schemes for the benjamin-bona-mahony (bbm) equation. Applied Mathematics and Computation, 2006, 182(2):999-1005.

[12] Kadri, T., Khiari, N., Abidi, F., and Omrani, K., Methods for the numerical solution of the benjamin-bona-mahony-burgers equation. Numerical Methods for Partial Differential Equations, 2008, 24(6):1501-1516.

[13] Dogan, A., Numerical solution of rlw equation using linear finite elements within galerkin's method. Applied Mathematical Modelling, 2002, 26(7):771-783.

[14] Qin, Y., Kong, H., Luo, D., and Feng, M., A fully discrete mixed finite elemen$\mathrm{t}$ method for the benjamin-bona-mahony(bbm) equation. Acta Mathematicae Applicatae Sinica, 2015.

[15] Quarteroni, A. M., and Valli, A., Numerical Approximation of Partial Differential Equations. Springer-Verlag, 1998. 